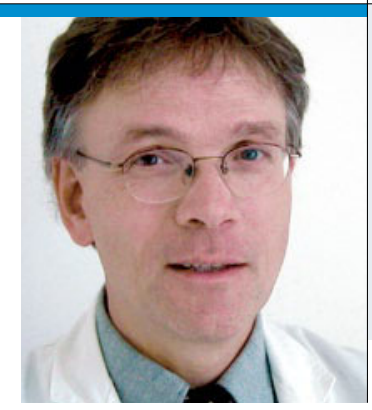

\title{
Fundstücke
}

Dr. med. Ulrich Mutschler, Chefredakteur

\section{Gekaufte Kongresse?}

Sollen die Organisation und die Referenten bei ärztlichen Fortbildungsveranstaltungen weiter von der Industrie unterstützt werden? In den USA wurden dazu die Teilnehmer bei eintägigen Fortbildungsveranstaltungen befragt [Tabas JA et al. Arch Intern Med 2011; 171: 840-6]: Sie gaben an, dass nur bei $7 \%$ der Veranstaltungen, die allein von den Teilnehmern finanziert werden, mit vorgefassten Urteilen zu rechnen sei. Diese Rate steigt von 46 über 80 auf $86 \%$, wenn die Fortbildungen zu 20, 60 bzw. $100 \%$ von einem einzigen

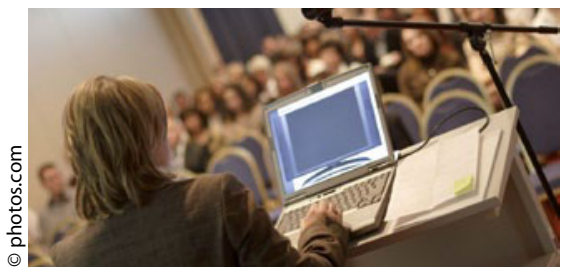

Sponsor gefördert werden. Bei über der Hälfte von Referenten, die für ihre Vorträge Geld erhalten, wird Voreingenommenheit unterstellt. Jedoch: Nur $42 \%$ wären bereit, eine höhere Teilnahmegebühr zu bezahlen.

\section{Erfolgreiche Hepatitis-B-Impfung}

Vor der Einführung flächendeckender Impfungen hatte die Urbevölkerung in Alaska die höchste Hepatitis-B-Rate der gesamten USA. Den positiven Effekt der 1984 begonnenen Impfungen konnten jetzt Kollegen vom Alaska Native Medical Center in Anchorage feststellen [McMahon $\mathrm{BJ}$ et al. Hepatology 2011 May 26. (Epub ahead of print)]. Im Surveillance-Register von 1981/82 wurden bei den unter 20-Jährigen 19 Fälle pro 100.000 Einwohner gezählt. Zwölf Jahre später hatte keiner der jungen Ureinwohner in Alaska eine akute Hepatitis B; die letzte akute Infektion ereignete sich 1992. Auch das

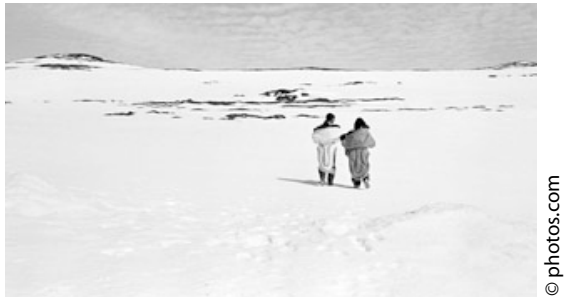

hepatozelluläre Karzinom in dieser Altersgruppe trat zuletzt 1999 auf (zwischen 1984 und 1988 drei Fälle, zwischen 1995 und 1999 keiner). Ebenso ging die Anzahl von jungen Menschen mit positivem Nachweis von HbsAg von 657 im Jahr 1987 auf zwei 2008 zurück, obwohl sich die Anzahl der Ureinwohner Alaskas von 75.000 auf 130.000 Einwohner erhöhte.

\section{Wie krank macht das Zahnen?}

Früher hießen Fieberkrämpfe gelegentlich auch „Zahnkrämpfe“. Auch heute noch werden viele andere Symptome auf das Zahnen zurückgeführt. Welche nun genau mit dem Zahnen zusammenhängen, untersuchten Kinderärzte aus Brasilien [Ramos-Jorge J et al. Pediatrics 2011; Aug 8. (Epub ahead of print)]. Sie dokumentierten über acht Monate den Gesundheitszustand von 47 zahnenden Kleinkindern. Täglich wurde der Zahnstatus untersucht, die Temperatur gemessen und die Mütter nach bestimmten Symptomen befragt. Die Kinder im Alter von fünf bis 15 Monaten bekamen insgesamt 231 Zähne. Der Zahndurchbruch war verbunden mit einem Anstieg der Körpertemperatur im Mittel um $0,12^{\circ} \mathrm{C}$ (im Ohr). Außerdem traten viele Symptome häufiger am Tag des Zahndurchbruchs und am Tag danach auf: v. a. Reizbarkeit, vermehrter Speichelfluss, laufende Nase und
Appetitlosigkeit, etwas seltener Schlafstörungen, Hautausschläge und Diarrhö. Kein Kind zeigte Krämpfe oder Koliken, nur ganz selten wurde über Erbrechen berichtet. Als Fazit erklären daher die Autoren:„Wenn Kinder schwerwiegende Befunde oder Beschwerden haben, dann nicht vorschnell das Zahnen dafür verantwortlich machen, sondern nach anderen Ursachen suchen."

\section{Wirkung von Zink bei Otitis media fraglich}

Zur Prophylaxe von grippalen Infekten wird immer wieder Zink beworben. Zur Klärung, ob Zink auch einer Otitis media vorbeugen kann, wurde kürzlich ein Cochrane-Review über die Einnahme von Zink erstellt [Abba K et al. Cochrane Database Syst Rev. 2010; 2: CD006639]. Insgesamt zwölf Studien bei Kin-

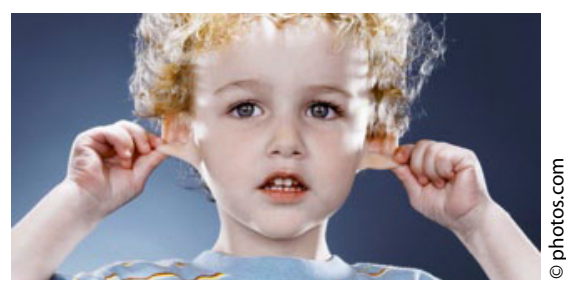

dern unter sechs Jahren aus zehn verschiedenen Ländern mit niedrigem und mittlerem Einkommen wurden analysiert. Bei gesunden Kindern aus schlechteren Verhältnissen zeigten zwei Untersuchungen keinen signifikanten Unterschied zwischen einer Zinksupplementation oder Placebo (3.191 Probanden). Dagegen kam in einer anderen Studie eine niedrigere Inzidenzrate für die Otitis in der Verum-Gruppe heraus (1.621 Probanden). Eine kleine Gruppe von 39 Kindern, die wegen starker Unterernährung untersucht wurde, profitierte ebenfalls von einer Zinkzufuhr. Die Autoren folgern, dass kein klarer Beweis für die Schutzwirkung von Zink gegenüber einer Otitis media bei sonst gesunden Kindern vorliegt; jedoch profitieren dystrophe Kinder durchaus. 